\section{Lipidmetabolismus und Insulinresistenz bei depressiven Patienten}

Daniel Kopf und Michael Deuschle Zentralinstitut für Seelische Gesundheit, Mannheim

psychoneuro 2005; 31 (4): 212-216

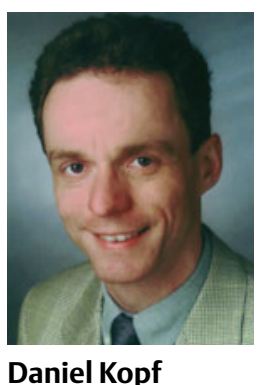

Daniel Kopf
Depression ist mit einem erhöhten kardiovaskulären Risiko verbunden. Hierzu trägt die Tatsache bei, dass depressive Patienten häufiger als die Allgemeinbevölkerung von einem metabolischen Syndrom betroffen sind, bei dem Diabetes mellitus Typ 2, bauchbetonte Adipositas, Hypertonie und/oder eine Dyslipidämie mit einer Insulinresistenz verknüpft sind. Cholesterin ist dagegen paradoxerweise in manchen Studien an depressiven Patienten erniedrigt. Unsere Daten zeigen erstmals einen Zusammenhang zwischen erniedrigtem Cholesterin und dem Hypercortisolismus Möglicherweise ist erniedrigtes Cholesterin ein Kennzeichen des melancholischen, hypercortisolämischen Subtyps der Depression. Klassische Antidepressiva wie das von uns untersuchte Amitriptylin und der SSRI Paroxetin zeigen einen neutralen oder sogar günstigen Einfluss auf Insulinresistenz und die Architektur der Lipoproteine. Damit kann eine medikamentöse antidepressive Therapie vermutlich dazu beitragen, auch das erhöhte kardiovaskuläre Risiko zu senken.

V erschiedene prospektive Studien zeigen, dass depressive Erkrankungen das Risiko einer kardiovaskulären Erkrankung erhöhen (14). Dabei liegt das relative Risiko im Vergleich zur Normalbevölkerung im Mittel der Studien etwa bei 1,5 bis 2 . Verschiedene Ursachen wurden hierfür in Betracht gezogen:

- zentralnervöse Störungen in der Regulation der Stresssysteme

- ungünstige Verhaltensweisen wie zum Beispiel Bewegungsarmut

- die Häufung klassischer kardiovaskulärer Risikofaktoren, wie sie zum Beispiel beim metabolischen Syndrom vorkommt (15).

Betrachtet man die einzelnen klassischen Risikofaktoren bei depressiven Patienten, so fällt auf, dass einer der wichtigsten kardiovaskulären Risikofaktoren, nämlich ein hoher Cholesterinspiegel, bei depressiven Patienten einigen Studien zufolge paradoxerweise vermindert zu sein scheint $(3,20,23)$.

Der vorliegende Artikel befasst sich mit den pathophysiologischen Zusammenhängen zwischen depressiven Erkrankungen, den Risikofaktoren des metabolischen Syndroms und dem Lipidstoffwechsel.

\section{Depression, Insulin- resistenz und das metabolische Syndrom}

Dass das metabolische Syndrom und die Depression eng verknüpft sind, zeigt eine prospektive epidemiologische Studie: Patienten mit Risiko, in der Zukunft ein metabolieiner Depression haben ein erhöhtes sches Syndrom zu entwickeln. Gleichzeitig haben Patienten mit einem metabolischen Syndrom ein erhöhtes Risiko, in der Zukunft an einer Depression zu erkranken (21).

Das metabolische Syndrom ist definiert als das Zusammentreffen von mindestens drei der in Tabelle 1 genannten Faktoren (19). Es fällt auf, dass die Grenzen hier sehr streng gefasst sind, so dass jeder dieser Faktoren für sich alleine noch keinen pathologischen Befund darstellt. Die klinische Bedeutung dieses Syndroms liegt vor allem darin, dass sich die verschiedenen Risikofaktoren gegenseitig verstärken. In der Botnia-Studie beispielsweise, einer prospektiven epidemiologischen Studie in Finnland und Schweden, lag das Risiko, an einer koronaren Herzkrankheit, einem Myokardinfarkt oder einem Schlaganfall zu leiden, bei Patienten mit einem metabolischen Syndrom dreimal so hoch wie in der Kontrollbevölkerung $(9,11)$

Pathophysiologisch im Zentrum des metabolischen Syndroms steht die Insulinresistenz, d.h. das verminderte Ansprechen der Zielzellen auf die blutzuckersenkende Wirkung von Insulin. Für jede einzelne Störung des metabolischen Syndroms konnte gezeigt werden, dass sie mit einer reduzierten Insulin- 
sensitivität, d.h. mit Insulinresistenz verknüpft ist $(9,13)$.

Eine Insulinresistenz wurde auch bei Patienten mit einer mittelschweren oder schweren depressiven Episode nachgewiesen (23). Entsprechend findet man bei Patienten mit depressiven Syndromen ein höheres Risiko, einen Diabetes mellitus Typ $2 \mathrm{zu}$ entwickeln (7), eine abdominelle Adipositas (25) und Störungen der Blutdruckregulation, insbesondere bei hypercortisolämischen depressiven Patienten (16).

Eine Insulinresistenz und damit ein metabolisches Syndrom können entstehen:

- durch genetische Faktoren

- durch Verhaltensfaktoren wie Bewegungsarmut, fettreiche Ernährung oder Rauchen sowie

- sekundär durch Entzündungskonstellationen oder Hormonstörungen.

Bei depressiven Erkrankungen wurde gezeigt, dass nichtgenetische Faktoren wie Bewegungsmangel oder Fehlernährung zu metabolischen Veränderungen beitragen können (18). Daneben ist die Depression bei einer beträchtlichen Subgruppe durch einen Hypercortisolismus gekennzeichnet (10). Da Kortisol auf den Glukosestoffwechsel antagonistisch zu Insulin wirkt, ist anzunehmen, dass der Hypercortisolismus auch zum metaboli- schen Syndrom bei Insulinresistenz beiträgt.

\section{Depression und \\ Fettstoffwechsel}

Die Daten zu Cholesterin und Depression sind teilweise widersprüchlich und lückenhaft. Ältere Interventionsstudien mit Lipidsenkern, bei denen nichtnatürliche Todesfälle, insbesondere Suizide, unter Intervention gehäuft auftraten, haben nahegelegt, dass niedrige Cholesterinspiegel mit depressiven Symptomen assoziiert ist (23). Damit in Einklang wurde berichtet, dass stationär behandelte depressive Patienten einen erniedrigten Cholesterinspiegel haben $(3,20)$. Vor kurzem wurde dieser Zusammenhang auch in einer prospektiven Studie bei älteren Menschen und in einer Studie an hochbetagten Menschen gefunden $(1,5)$. Als Mechanismus für diesen Zusammenhang wird angegeben, dass niedriges Cholesterin die Verfügbarkeit von Serotonin ungünstig beeinflusst (5). Somit wäre ein kausaler Mechanismus anzunehmen, der von niedrigem Cholesterin zu Depressivität führt.

Andere Daten legen allerdings nahe, dass Patienten sich bereits im Vorfeld der Depression schlechter ernähren und daher einen niedrigeren Cholesterinspiegel aufweisen (4).

Entsprechend wurden in neueren Interventionsstudien zur Cho-

\section{Abb. 1 Depression und kardiovaskuläres Risiko}

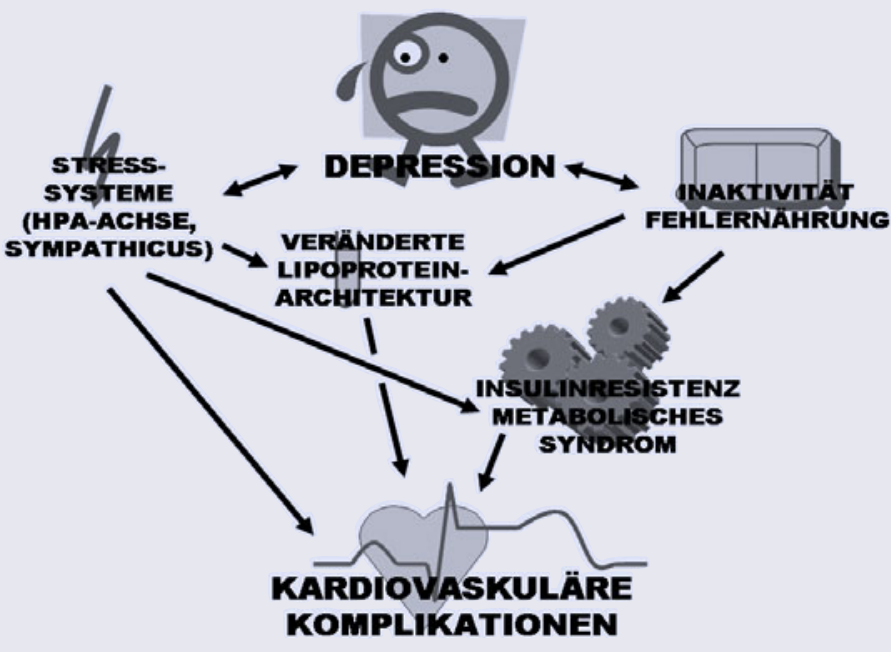

Vereinfachte schematische Darstellung des Zusammenhangs zwischen Depression und kardiovaskulärem Risiko. Erklärung siehe Text
Tab. 1 NCEP-Definition des metabolischen Syndroms

Bauchbetonte Adipositas: Bauchumfang

- Frauen $>88 \mathrm{~cm}$

- Männer $>102 \mathrm{~cm}$

Triglyzeride $\quad \geq 150 \mathrm{mg} / \mathrm{dl}(>2,7 \mathrm{mmol} / \mathrm{l})$

HDL-Cholesterin

$\begin{array}{ll}\text { - Frauen } & <50 \mathrm{mg} / \mathrm{dl}(<1,3 \mathrm{mmol} / \mathrm{l}) \\ \text { - Männer } & <40 \mathrm{mg} / \mathrm{dl}(<1,05 \mathrm{mmol} / \mathrm{l}) \\ \text { Blutdruck } & \geq 130 / \geq 85 \mathrm{mmHg} \\ \text { Nüchternglukose } & \geq 110 \mathrm{mg} / \mathrm{dl}(\geq 6,1 \mathrm{mmol} / \mathrm{l})\end{array}$

Ein metabolisches Syndrom liegt vor, wenn mindestens drei der genannten Kriterien erfüllt sind (19)

lesterinsenkung mit Statinen keine erhöhten Raten an Depressivität mehr gefunden (27). Genauere Analysen der älteren Interventionsstudien ergaben, dass die erhöhten Suizidraten unter Lipidsenkern durch statistische Artefakte bedingt waren. So waren beispielsweise in der Interventionsgruppe mehr Patienten mit Alkoholabhängigkeit (25).

Die größte vorliegende Querschnittsstudie zu dieser Frage wurde im Rahmen der „National Health and Nutrition Survey Examination“" in den USA durchgeführt und untersuchte die Lebenszeitprävalenz der Depression an knapp 7000 Menschen zwischen 18 und 39 Jahren. In dieser Studie fand sich kein Unterschied im Cholesterinspiegel zwischen Patienten mit oder ohne durchgemachte depressive Episode (8).

Ein Problem dieser großen Studien ist, dass sie nur unzureichend die Subfraktionen der Plasmalipide unterscheiden, die aber entscheidend die atherogene Wirkung von Cholesterin bedingen. Gesamtcholesterin besteht aus den atherogenen LDL- und den protektiven HDLPartikeln. In jüngerer Zeit wurde aber zunehmend die Bedeutung der Architektur der LDL-Partikel erkannt. Wenn die cholesterinreichen LDL-Partikel zusätzlich reich an Triglyzeriden werden, so werden sie dichter gepackt, dadurch kleiner und können leichter in der Gefäßwand stranden. Dort werden sie ebenfalls leichter oxidiert und lösen daher eine Entzündungsreaktion der Gefäßwand aus, die in Atherosklerose mündet. Kleine, triglyzeridreiche LDL-Partikel bedingen also ein be- 


\section{Abb. 2 Darstellung der Veränderungen}

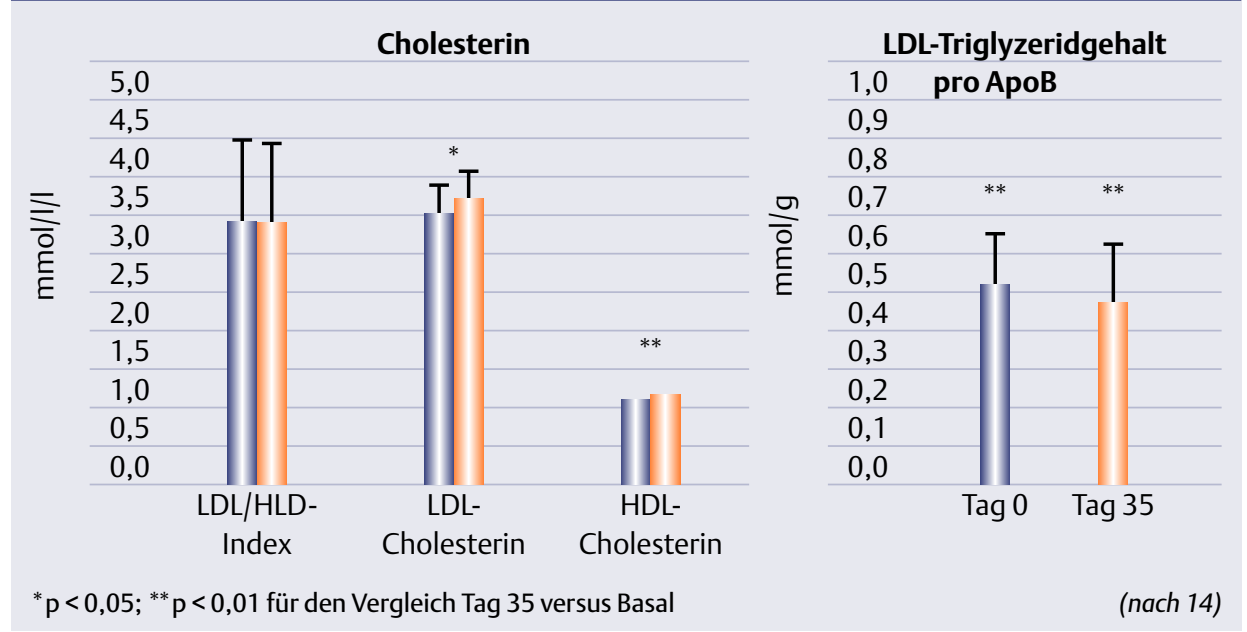

Veränderungen von LDL- und HDL-Cholesterin sowie des LDL/HDL-Quotienten $(A)$ und des Triglyzeridgehalts der LDL-Partikel (B) unter antidepressiver Therapie

sonders hohes Risiko atherosklerotischer Komplikationen und sind übrigens eng mit Insulinresistenz assoziiert (2). Bisher gibt es keine sorgfältigen Untersuchungen zur Architektur der Lipoproteine.

Bei der Widersprüchlichkeit epidemiologischer Daten wäre es sinnvoll, Subgruppen genauer zu untersuchen. Möglicherweise gibt es bedeutsame Unterschiede zwischen jüngeren und älteren depressiven Patienten, wie die oben genannten Studien vermuten lassen. Ein weiterer entscheidender Unterschied könnte im Subtyp der Depression liegen: So könnte der melancholische Subtyp, der mit einem anderen Gewichtsund Ernährungsverhalten als die atypische Depression einhergeht, auch Unterschiede bezüglich des Lipidstoffwechsels ergeben. Geschlecht und Menopausenstatus sind ebenfalls potentiell konfundierende Variablen. Allerdings sind solche Studien in ausreichender Fallzahl schwierig durchzuführen, da sie sowohl eine sorgfältige psychiatrische Charakterisierung als auch genaue metabolische Analysen erfordern, die in großen Patientenkollektiven schwer durchzuführen sind.

Medikamentöse Modulation metabolischer Parameter

Eine andere Möglichkeit, die Zusammenhänge etwas besser zu verstehen, ist die gezielte pharmakologische Intervention. In einer Studie an
78 Patienten mit „major depression“ nach DSM IV haben wir uns den differentiellen Effekt von Paroxetin und Amitriptylin auf das Körpergewicht und auf den Hypercortisolismus zunutze gemacht, um den Einfluss von Insulinsensitivität und Kortisol auf den Lipidstoffwechsel zu untersuchen (14). Die Therapie mit trizyklischen Antidepressiva wie Amitriptylin führt zu einer Gewichtszunahme und gleichzeitig zu einer Normalisierung der erhöhten Aktivität der Hypothalamus-Hypophysen-Nebennierenrinden-Achse (HPA-Achse), gemessen am Speichelkortisol. Im Gegensatz dazu bleibt das Gewicht konstant oder sinkt leicht unter Therapie mit dem selektiven Serotonin-Wiederaufnahmehemmer (SSRI) Paroxetin. Die Hypercortisolämie wird nicht relevant beeinflusst (6). Damit können zwei wichtige Parameter für die Regulation metabolischer Faktoren das Körpergewicht und der Hypercortisolismus - differentiell beeinflusst werden.

Die Patienten erhielten nach einer Auswaschphase von sechs Tagen eine Therapie mit Amitriptylin oder Paroxetin über fünf Wochen. Vor Beginn und am Ende der medikamentösen Therapie wurde ein ausführliches Lipidprofil, das auch die Architektur der Lipoproteine erfasst, bestimmt. Weiterhin waren Insulinsensitivität, Kortisol und das Ansprechen auf die Therapie im Fremdbeurteilungsbogen (Hamilton D) Zielparameter.
Insulinsensitivität und Lipidprofil bei depressiven Patienten

Wichtigste Determinante der Insulinsensitivität war bei normalgewichtigen Patienten die Aktivität der Hypothalamus-HypophysenNebennierenachse: Patienten mit hohem Speichelkortisol waren insulinresistenter.

Bei übergewichtigen Patienten zeigte sich ein weiterer interessanter Zusammenhang: Das Speichelkortisol korrelierte signifikant negativ mit dem Gesamtcholesterin, dem LDL-Cholesterin, mit Apolipoprotein $B$ und dem LDL-/HDL-CholesterinQuotienten.

Diese Daten bieten erstmals eine Erklärung für die epidemiologisch beobachtete Tendenz zu niedrigem Cholesterin bei Depression: Möglicherweise ist das niedrige Cholesterin eine Folge des Hypercortisolismus. Da nicht alle depressiven $\mathrm{Pa}$ tienten auch einen Hypercortisolismus aufweisen, sondern bevorzugt Patienten mit melancholischem Subtyp, können epidemiologische Studien variieren, je nachdem, wie hoch der Anteil atypischer Depressionen ist.

Der molekulare Mechanismus könnte darin liegen, dass Kortisol den intrazellulären Rezeptor PPAR alpha stimuliert, der wiederum eine zentrale Rolle in der Synthese der Lipoproteine spielt (17).

\section{Einflüsse medikamentöser antidepressiver Therapie ... ... auf Kortisol}

Von den 34 Patienten, die Amitriptylin erhielten, sprachen 24 auf die Therapie an; von den 44 Patienten unter Paroxetin zeigten 26 klinisches Ansprechen.

Die Speichelkortisolkonzentrationen blieben konstant in der mit Paroxetin behandelten Gruppe, fielen dagegen signifikant ab bei Patienten, die auf eine Therapie mit Amitriptylin ansprachen.

...auf Insulinsensitivität, Körpergewicht und triglyzeridreiche Lipoproteine

Patienten, die auf Amitriptylin ansprachen, nahmen signifikant an Gewicht zu. Das Gewicht bei allen Patienten, die Paroxetin erhielten, 
und bei Patienten, die auf Amitriptylin nicht ansprachen, blieb konstant.

Insulinsensitivität und C-PeptidSpiegel blieben konstant, unabhängig vom eingesetzten Antidepressivum, wahrscheinlich weil sich die gegenläufigen Effekte auf Kortisol und auf das Körpergewicht ausglichen. Die Glukosewerte im Glukosetoleranztest besserten sich bei allen Respondern. Beide Therapiestrategien sind also, was die Insulinsensitivität angeht, gleichwertig.

Parallel zum Gewicht verhielten sich die Gesamttriglyzeride und das Apolipoprotein E: Bei Respondern auf Amitriptylin stiegen die Werte an, bei allen anderen Gruppen blieben sie gleich.

\section{...auf cholesterinreiche Lipo- proteine}

Bei den cholesterinreichen Lipoproteinen gab es keine signifikanten Unterschiede zwischen den mit Amitriptylin und den mit Paroxetin behandelten Gruppen. Auch das klinische Ansprechen hatte keinen statistisch signifikanten Einfluss auf die Veränderungen.

Allerdings fanden sich kleine, in allen Gruppen parallel laufende Veränderungen. Für das Gesamtcholesterin zeigte sich zwar nur ein nicht signifikanter Trend zu einem Anstieg nach Therapie. Bei beiden großen Unterfraktionen von Cholesterin, HDL und LDL, waren diese Anstiege jedoch signifikant (HDL-Cholesterin $\mathrm{p}<0,01$, LDL-Cholesterin $\mathrm{p}<0,05$, Abb. 2). Da hierbei HDL etwas stärker anstieg als LDL, resultierte ein leicht verbesserter HDL-/LDL-Quotient.

Gleichzeitig fand sich ein statistisch signifikanter Abfall des Triglyzeridanteils in LDL-Partikeln, ausgedrückt als der Quotient aus LDLTriglyzeriden und Apolipoprotein B. Das bedeutet, dass sich die Architektur der LDL-Partikel hin zu den günstigeren, triglyzeridärmeren großen Partikeln verändert.

Damit verändern sich also die Gesamtcholesterinkonzentrationen unter antidepressiver Therapie zwar kaum. Die Zusammensetzung und Architektur der Lipoproteine werden aber günstig beeinflusst, so dass ein geringeres kardiovaskuläres Risiko folgt.

\section{Zusammenfassung}

Depressionen sind mit einem erhöhten kardiovaskulären Risiko und folglich erhöhter kardiovaskulärer Mortalität verknüpft. $\mathrm{Zu}$ diesem erhöhten Risiko trägt die Tatsache bei, dass depressive Patienten häufiger als die Allgemeinbevölkerung von einem metabolischen Syndrom betroffen sind, einem Zusammentreffen von Risikofaktoren, in deren Zentrum die Insulinresistenz steht.

Zum Cholesterinstoffwechsel, einem der wichtigsten Risikofaktoren, sind die Daten bei depressiven Patienten widersprüchlich. Teilweise sprechen die Studien sogar für ein vermindertes Cholesterin bei depressiven Patienten. Studien in genau definierten Subgruppen, z.B. bezüglich des Alters, des Subtyps der Depression oder des Geschlechts sind wünschenswert, um diese $\mathrm{Zu}$ sammenhänge genauer zu verstehen. Unsere eigenen Daten zeigen erstmals einen Zusammenhang zwischen erniedrigten Cholesterinspiegeln und der Hypercortisolämie.

Wegen des beschriebenen kardiovaskulären Risikos ist es besonders wichtig, zu überprüfen, wie sich eine medikamentöse Therapie auf kardiovaskuläre Risikofaktoren auswirkt. Unsere Daten zeigen, dass auch Trizyklika trotz der Gewichtszunahme keine Verschlechterung der Insulinsensitivität und des Cholesterinstoffwechsels zur Folge haben, da sie gleichzeitig die Hypercortisolämie bessern. Lediglich die Triglyzeride scheinen bei Respondern auf Trizyklika leicht anzusteigen. Bezüglich des Cholesterinstoffwechsels ist festzustellen, dass sowohl das trizyklische Antidepressivum Amitriptylin als auch der selektive Serotonin-Wiederaufnahmehemmer (SSRI) Paroxetin die Architektur der Lipoproteine eher günstig beeinflussen.

\section{Lipid metabolismus and insulin resistance in depressive patients} Major depression conveys an increased cardiovascular risk. This is in part due to a higher prevalence of the metabolic syndrome in depressed patients, when compared to the general population. The metabolic syndrome is defined as an association of type- 2 diabetes mellitus, visceral obesity, hypertension and dyslipidemia with insulin resistance. Paradoxically, depression has been linked to low cholesterol by some studies. We found a negative correlation between cortisol and cholesterol in a subgroup of depressed patients, suggesting that low cholesterol may be a feature of the melancholic, hypercortisolemic subtype of depression. Classical antidepressant drugs such as amitriptyline and SSRI such as paroxetin have neutral or slightly beneficial effects on insulin resistance and architecture of lipoproteins. Therefore, pharmacological treatment of depression may help lowering the increased cardiovascular risk.

\section{Key Words \\ depression - cholesterol - insulin resistance - metabolic syndrome}

\section{Literatur}

1. Äijänseppä S, Kivinen P, Helkala EL, Kivelä SL, Tuomilehto J, Nissinen A. Serum cholesterol and depressive symptoms in elderly Finnish men. Int J Geriatr Psychiatry 2002; 17: 629-634

2. Ambrosch A, Mühlen I, Kopf D, Augustin W, Dierkes ], König W, Luley C, Lehnert H. LDL size distribution in relation to insulin sensitivity and lipoprotein pattern in young and healthy subjects. Diab Care 1998; 21 : 2077-2084

3. Borgherini G, Dorz S, Conforti D, Scarso C, Magni G. Serum cholesterol and psychological distress in hospitalized depressed patients. Acta Psychiatr Scand 2002; 105: 149-152

4. Boston PF, Dursun SM, Reveley MA. Cholesterol and mental disorder. Br J Psychiatry 1996; 169: 682-689

5. Cervilla J, Prince M, Rabe-Hesketh S. Vascular disease risk factors as determinants of incident depressive symptoms: a prospective community-based study. Psychol Med 2004; 34: 635-641

6. Deuschle M, Hamann B, Meichel C, Krumm B, Lederbogen F, Kniest A, Colla M, Heuser I. Antidepressive treatment with amitriptyline and paroxetine: effects upon saliva cortisol concentrations. J Clin Psychopharmacol 2003; 23: 20-25

7. Eaton WW, Armenian H, Gallo J, Pratt L, Ford DE. Depression and risk for onset of type II diabetes. A prospective populationbased study. Diabetes Care 1996; 19: 1097102

8. Ford DE, Erlinger TP. Depression and CReactive Protein in US Adults. Data From the Third National Health and Nutrition Examination Survey. Arch Intern Med 2004; 164 : 1010-1014

9. Groop L, Orho-Melander M. The dysmetabolic syndrome. J Intern Med 2001; 250: 105-20 
10. Holsboer $F$. The corticosteroid receptor hypothesis of depression. Neuropsychopharmacology 2000; 23: 477-501

11. Isomaa B, Almgren P, Tuomi T, Forsen B, Lahti K, Nissen M, Taskinen MR, Groop L. Cardiovascular morbidity and mortality associated with the metabolic syndrome. Diabetes Care 2001; 24: 683-9

12. Katz A, Nambi SS, Mather K et al. Quantitative insulin sensitivity check index: a simple, accurate method for assessing insulin sensitivity in humans. J Clin Endocrinol Metab 2000; 85: 2402-10

13. Kopf $D$, Lehnert $H$. Predictive examinations of phenotypically healthy offspring of patients with a metabolic syndrome. Dtsch Med Wochenschr 2001; 126: 235-40

14. Kopf D, Westphal S, Luley C, Ritter S Gilles M, Weber-Hamann B, Lederbogen F, Lehnert $H$, Henn FA, Heuser I, Deuschle M. Lipid metabolism and insulin resistance in depressed patients. Significance of weight. hypercortisolism, and antidepressant treatment. J Clin Psychopharmacol 2004; 24: 527-531

15. Lederbogen F, Deuschle M, Heuser I. Depression - ein kardiovaskulärer Risikofaktor. Internist 1999; 40: 1119-1121

16. Lederbogen F, Gernoth C, Hamann B, Kniest A, Heuser I, Deuschle M. Circadian blood pressure regulation in hospitalized depressed patients and non-depressed comparison subjects. Blood Press Monit 2003; 8: 71-6

17. Lemberger T, Saladin R, Vazquez M, Assimacopoulos F, Staels B, Desvergne B, Wahli W, Auwerx J. Expression of the peroxisome proliferator-activated receptor $\alpha$ gene is stimulated by stress and follows a diurnal rhythm. J Biol Chem 1996; 271: 1764-1769

18. McCaffery JM, Niaura R, Todaro JF, Swan GE, Carmelli D. Depressive Symptoms and Metabolic Risk in Adult Male Twins Enrolled in the National Heart, Lung, and Blood Institute Twin Study. Psychosom Med 2003; 65: 490-497

19. NCEP: National Cholesterol Education Program. Executive Summary of The Third Report of The National Cholesterol Education Program (NCEP) Expert Panel on Detection, Evaluation, And Treatment of High Blood Cholesterol In Adults (Adult Treatment Panel III). JAMA 2001; 285: 24862497

20. Olusi SO, Fido AA. Serum lipid concentrations in patients with major depressive disorder. Biol Psychiatry. 1996; 40: 1128 1131

21. Raikkonen K, Matthews KA, Kuller LH. The relationship between psychological risk attributes and the metabolic syndrome in healthy women: antecedent or consequence? Metabolism 2002; 51: 1573-1577 22. Thomas AJ, Kalaria RN, O'Brien JT.
Depression and vascular disease: what is the relationship? J Affect Disorders 2004; 79: 81-95

23. Wardle J. Cholesterol and psychological well-being. J Psychosom Res 1995; 39: 549562

24. Weber B, Deuschle M, Schweiger U, Körner A, Standhardt H, Schmider J, Heuser I. Major depression and impaired glucose tolerance. Exp Clin Endocrinol Diabet 2000; 108: $187-190$

25. Weber-Hamann B, Hentschel F, Kniest A, Deuschle M, Colla M, Lederbogen F, Heuser I. Hypercortisolemic depression is associated with increased intra-abdominal fat. Psychosom Med 2002; 64: 274-277

26. Wysowski DK, Gross TP. Deaths due to accidents and violence in two recent trials of cholesterol-lowering drugs. Arch Intern Med 1990; 150: 2169-2172

27. Young-Xu Y, Chan KA, Liao JK, Ravid S, Blatt CM. Long-term statin use and psychological well-being. J Am Coll Cardiol 2003; 42: 690-697

\section{Korrespondenzadresse:}

Dr. Daniel Kopf

Zentralinstitut für Seelische Gesundheit

Postfach 212120

68072 Mannheim

kopf@zi-mannheim.de 